\title{
Adverse Effect of the Methanotroph Methylocystis sp. M6 on the Non-Methylotroph Microbacterium sp. NM2
}

\author{
So-Yeon Jeong ${ }^{1}$, Kyung-Suk Cho ${ }^{2}$, and Tae Gwan Kim ${ }^{1 *}$ \\ ${ }^{1}$ Department of Microbiology, Pusan National University, Pusan 46241, Republic of Korea \\ ${ }^{2}$ Department of Environmental Science and Engineering, Ewha Womans University, Seoul 03760, Republic of Korea
}

\author{
Received: April 12, 2018 \\ Revised: July 19, 2018 \\ Accepted: August 23, 2018 \\ First published online \\ August 24, 2018 \\ *Corresponding author \\ Phone: +82-515102268; \\ Fax: +82-515141778; \\ E-mail: tkim@pusan.ac.kr \\ pISSN 1017-7825, eISSN 1738-8872 \\ Copyright@ 2018 by \\ The Korean Society for Microbiology \\ and Biotechnology
}

\begin{abstract}
Several non-methylotrophic bacteria have been reported to improve the growth and activity of methanotrophs; however, their interactions remain to be elucidated. We investigated the interaction between Methylocystis sp. M6 and Microbacterium sp. NM2. A batch co-culture experiment showed that NM2 markedly increased the biomass and methane removal of M6. qPCR analysis revealed that NM2 enhanced both the growth and methane-monooxygenase gene expression of M6. A fed-batch experiment showed that co-culture was more efficient in removing methane than M6 alone (28.4 vs. $18.8 \mu \mathrm{mol} \cdot \mathrm{l}^{-1} \cdot \mathrm{d}^{-1}$ ), although the biomass levels were similar. A starvation experiment for 21 days showed that M6 population remained stable while NM2 population decreased by $66 \%$ in co-culture, but the results were opposite in pure cultures, indicating that M6 may cross-feed growth substrates from NM2. These results indicate that M6 apparently had no negative effect on NM2 when M6 actively proliferated with methane. Interestingly, a batch experiment involving a dialysis membrane indicates that physical proximity between NM2 and M6 is required for such biomass and methane removal enhancement. Collectively, the observed interaction is beneficial to the methanotroph but adversely affects the non-methylotroph; moreover, it requires physical proximity, suggesting a tight association between methanotrophs and non-methylotrophs in natural environments.
\end{abstract}

Keywords: Methylocystis, Microbacterium, microbial interaction, physical proximity

\section{Introduction}

Methanotrophs, defined as aerobic methane-oxidizing bacteria, utilize methane as a sole carbon and energy source; thus, they have an important role in the global carbon cycle $[1,2]$. Methane monooxygenase (MMO) is the key enzyme for acting as a methanotroph because it oxidizes methane to methanol. Due to its low specificity, MMO can oxidize different recalcitrant compounds such as alkanes and aromatic hydrocarbons [3-5]. Therefore, methanotrophs are broadly used as biocatalysts in many biotechnological processes, such as in biodegradation systems for methane as a greenhouse gas and recalcitrant contaminants $[6,7]$. Most methanotrophs have been considered to be obligate to methane, but some are facultative methanotrophs that can utilize multi-carbon compounds including ethanol and organic acids, such as acetate, pyruvate, and malate [8,9].
Methanotrophs have been commonly encountered in natural environments such as oceans, fresh waters, wetlands, and soils [1, 2, 9]. They have been reported to be closely associated with various organisms such as plants, protists, and marine invertebrates as well as with other bacteria $[1,10,11]$. For instance, methanotrophs have synergistic interactions with sphagnum mosses and microalgae by exchanging $\mathrm{CO}_{2}$ and $\mathrm{O}_{2}$ [12-14]. They are reported to be endosymbionts of marine invertebrates [10], and also prey for bacterivores [15]. Therefore, it is probable that methanotrophs significantly interact with many other biotic components in different ways.

There are different types of microbial interactions, some of which can be beneficial to at least one participant, e.g., commensalism, synergism, mutualism, parasitism, and predation [16]. Recent reports indicate that methanotrophic activity can be enhanced by commensalism and synergism 
via a metabolite exchange between methanotrophic and non-methylotrophic bacteria [17-20]. For example, methanotrophs have been shown to have a metabolic association with heterotrophic bacteria [19]. Metabolites from nonmethylotrophic bacteria have been shown to support the activity and growth of co-cultured methanotrophs [17, 18]. The metabolite cobalamin from a Rhizobium isolate, as well as riboflavin and organic acids, can stimulate methanotrophic activity. In contrast, methanol use by a methylotroph, i.e., cross-feeding, can be beneficial to methanotrophs because excess methanol generated during the methane oxidation process is toxic [20]. To the best of our knowledge, methanotrophs have not been reported to take advantage of other bacteria via mutualism, parasitism, and predation, which require intimate physical contact.

The main purpose of this study was to investigate and identify the interaction between Microbacterium sp. NM2 as a non-methylotroph and Methylocystis sp. M6 as a methanotroph. We tested whether co-cultivation of NM2 with M6 can increase the methanotrophic activity and population growth of M6, and whether a physical interaction is responsible for such a partnership.

\section{Materials and Methods}

\section{Organisms}

Microbacterium sp. NM2, Methylocystis sp. M6, Methylosinus trichosporium $\mathrm{OB} 3 \mathrm{~b}$, and 2 methanotrophic consortia were used in this study. NM2 was isolated from the Methylocystis-dominant methanotrophic consortium originating from soil [21]. This consortium was serially diluted with a sterile $0.9 \% \mathrm{NaCl}$ solution and spread on Difco R2A agar (BD Diagnostics, Sparks, USA) plates. A pure colony of NM2, yellow-pigmented, was obtained by subsequent transfers (more than three) to new R2A agar plates and maintained in R2A agar medium. To identify NM2, the 16S rRNA gene was amplified using the primer pair 341f (5'-CCTACG GGAGGCAGCAG-3') and 907r (5'-CCGTCAATTCCTTTRAGTTT$\left.3^{\prime}\right)$. The partial sequence of the $16 \mathrm{~S}$ rRNA gene was compared with known DNA sequences using the Basic Local Alignment Search Tool (BLAST) (http://blast.ncbi.nlm.nih.gov). The sequence obtained in this study was deposited in the DNA data bank of Japan database (http://www.ddbj.nig.ac.jp) under the accession number LC348727. NM2 was identified as a Microbacterium species (phylum Actinomycetes), and it has been deposited in the Korean collection for type cultures (http://kctc.kribb.re.kr) (world data center for microorganisms, WDCM597) under the collection number KCTC 29496. It was tested whether NM2 utilizes methane, methanol, and formate in nitrate mineral salts (NMS) medium. NMS contained $\mathrm{MgSO}_{4} \cdot 7 \mathrm{H}_{2} \mathrm{O} 1 \mathrm{~g} / \mathrm{l}, \mathrm{CaCl}_{2} \cdot 2 \mathrm{H}_{2} \mathrm{O}$ $0.134 \mathrm{~g} / \mathrm{l}, \mathrm{KNO}_{3} 1 \mathrm{~g} / \mathrm{l}, \mathrm{KH}_{2} \mathrm{PO}_{4} 0.272 \mathrm{~g} / 1, \mathrm{Na}_{2} \mathrm{HPO}_{4} \cdot 12 \mathrm{H}_{2} \mathrm{O} 0.717 \mathrm{~g} / 1$ [22]. NM2 was physiologically profiled using BIOLOG Ecoplates
(Biolog, Hayward, USA), which contain typical carbon sources for soil microorganisms.

Four methanotrophic cultures (two isolates and two consortia) were used in this study. Methylocystis sp. M6 (the collection no. KCTC 11519) [23] and Methylosinus trichosporium OB3b (KCTC 12568) were used as the methanotrophic isolates. Both of those isolates are reported to be obligately methanotrophic. In this study, we used M6 as a model methanotroph because M6 has shown the potential to interact with other non-methanotrophic bacteria [21]. The two methanotrophic consortia originated from riparian wetland soil at Gongju City, South Chungcheong Province, South Korea, and landfill soil at Gapyeong County, Gyeonggi Province, South Korea, and have been used in methane oxidation studies [24, 25]. They were designated as consortium A and consortium B, respectively. The methanotrophic cultures were maintained in NMS medium with 100,000 ppm methane as previously described by Jeong et al. [21]. $\mathrm{CuSO}_{4}$ was added to a final concentration of $30 \mu \mathrm{M}$ in order to support particulate $\mathrm{MMO}$ (pMMO) activity.

\section{Batch Co-Cultures of NM2 and Methanotrophs}

$\mathrm{NM} 2$ was grown in R2A broth at $30^{\circ} \mathrm{C}$ with an agitation of $200 \mathrm{rpm}$ for three days. Cells were harvested by centrifugation at $5,000 \times g$ for $10 \mathrm{~min}$ and washed with NMS. The washing step was repeated twice. The four methanotrophs were cultivated in NMS medium at $30^{\circ} \mathrm{C}$ with an agitation of $200 \mathrm{rpm}$ with $100,000 \mathrm{ppm}$ methane. The methanotrophic cultures were harvested by centrifugation at 5,000 $\times g$ for $10 \mathrm{~min}$ and washed with NMS once. To measure dry cell weight, the cell suspension was vacuumfiltrated through glass-fiber filter paper (Whatman 1825-047, UK) and dried in a dry oven at $105^{\circ} \mathrm{C}$ for $2 \mathrm{~h}$.

For each set of co-culture, NM2 and a methanotroph were independently prepared. Cell suspensions of each set were adjusted to the same concentration (a range of 0.4 to $0.9 \mathrm{~g} / \mathrm{l}$ ). After an overnight incubation at $4^{\circ} \mathrm{C}$ for normalizing each set, a methanotroph was mixed with either NM2 or fresh NMS medium at a ratio of 1:49 (v/v). Fifty $\mathrm{ml}$ of each mixture were placed in 600-ml serum bottles $(n=2)$, and the bottles were sealed with a butyl-rubber stopper and parafilm. Methane $(99.9 \%)$ was added to a final concentration of $100,000 \mathrm{ppm}$. They were incubated at $30^{\circ} \mathrm{C}$ with an agitation of $200 \mathrm{rpm}$ for 4 days. When the methane concentration was below 10,000 ppm, the serum bottles were aerated on a clean bench, and methane was spiked again into the bottles.

\section{Population Dynamics of NM2 and M6}

NM2 and M6 cells were prepared as described above. They were adjusted to a final concentration of $0.4 \mathrm{~g}$ dry cell $1 \mathrm{l}^{-1}$. M6 was mixed with either NM2 or fresh NMS medium at a ratio of 1:49. Fifty $\mathrm{ml}$ of each mixture were placed in $600-\mathrm{ml}$ serum bottles $(n=2)$. These bottles were then sealed with a butyl-rubber stopper and parafilm. Methane was added to a final concentration of $100,000 \mathrm{ppm}$. They were incubated at $30^{\circ} \mathrm{C}$ with an agitation of $200 \mathrm{rpm}$ for 21 days. Ten $\mathrm{ml}$ of each culture were replaced with 
fresh NMS every week. When methane concentration was below $10,000 \mathrm{ppm}$, the serum bottles were aerated on a clean bench, and methane was spiked again into the bottles. Samples were collected on days $0,7,14$, and 21 , and cells were harvested from $1 \mathrm{ml}$ of each sample by centrifugation at 16,000 $\times g$ for $5 \mathrm{~min}$. NM2 and M6 were quantified using quantitative real-time PCR (qPCR).

Another experiment investigated the effects of starvation on coculture of NM2 and M6. The NM2 and M6 cells were prepared in NMS as described above to a cell concentration of $0.4 \mathrm{~g} / 1$. M6 was mixed with either NM2 or fresh NMS medium at a ratio of 1:49. In addition, NM2 alone (no M6 added) was prepared. Fifty $\mathrm{ml}$ of each mixture were placed in $600-\mathrm{ml}$ serum bottles $(n=2)$. These bottles were then sealed with a butyl-rubber stopper and parafilm. The cultures were incubated at $30^{\circ} \mathrm{C}$ with an agitation of $200 \mathrm{rpm}$ for 21 days without methane. Samples were collected on days 0,7 , and 21 , and the cells were harvested from $1 \mathrm{ml}$ of each sample by centrifugation at $16,000 \times g$ for $5 \mathrm{~min}$. NM2 and M6 were quantified using qPCR.

\section{Physical Separation between NM2 and M6 Using a Dialysis Membrane}

A dialysis membrane was used to allow NM2 and M6 to interact through chemical compounds. NM2 and M6 cells were prepared as described above. M6 was diluted with fresh NMS medium at a ratio of 1:9. Fifty $\mathrm{ml}$ of NM2 were contained within a dialysis membrane tube ( $34 \mathrm{~mm}$ wide, $50 \mathrm{kDa}$ pore size; CelluSep, Membrane Filtration Products, INC, USA). The dialysis tube was placed in a 1-L media bottle, to which $50 \mathrm{ml}$ of the diluted M6 was added. M6 was mixed directly with either NM2 or fresh NMS medium at a mixing ratio of 1:9. One hundred $\mathrm{mL}$ of each mixture were placed in a 1-L media bottle. There were two replicates for each treatment. Bottles were sealed with a silicone rubber stopper. Methane was added to a final concentration of $100,000 \mathrm{ppm}$. The bottles were incubated at $30^{\circ} \mathrm{C}$ with an agitation of $200 \mathrm{rpm}$ for 4 days. When methane concentration was below 10,000 ppm, the serum bottles were aerated on a clean bench, and methane was spiked again into the bottles.

\section{Fed-Batch Co-Culture of NM2 and M6}

NM2 and M6 cells were prepared as described above. The cell mixtures were adjusted to a final concentration of $0.4 \mathrm{~g} / \mathrm{l}$. M6 was mixed with either NM2 or fresh NMS at a ratio of 1:49. Fifty $\mathrm{ml}$ of the mixture were placed in 600-ml serum bottles. The bottles were then sealed with a butyl-rubber stopper and parafilm. Methane was added to a final concentration of $100,000 \mathrm{ppm}$. This was incubated at $30^{\circ} \mathrm{C}$ with an agitation of $200 \mathrm{rpm}$. The co-culture was acclimated for up to 6 days, and then operated with a hydraulic retention time (HRT) of 5 days for a period of 16 days as a fed-batch process ( $10 \mathrm{ml}$ refreshed every day). The bottles were aerated on a clean bench, and methane was added to a concentration of 100,000 ppm every day. Methane concentrations and biomass were monitored, and samples were collected on days $0,6,13,18$, and 22 . Harvested cells were frozen at $-70^{\circ} \mathrm{C}$ prior to use.

\section{Gas Analysis}

Methane and carbon dioxide concentrations were monitored using gas chromatography (GC; 6850N, Agilent Technologies, USA or GC-2010 plus, Shimadzu, Japan). The Agilent GC device was equipped with a wax column $(30 \mathrm{~m} \times 0.32 \mathrm{~mm} \times 0.25 \mu \mathrm{m}$, Supelco, USA) and a flame ionization detector. The oven, injector, and detector temperatures were set at $100^{\circ} \mathrm{C}, 230^{\circ} \mathrm{C}$, and $230^{\circ} \mathrm{C}$, respectively, with $\mathrm{N}_{2}$ as the carrier gas. The Shimadzu GC device was equipped with a Rt-Q-BOND column $(30 \mathrm{~m} \times 0.53 \mathrm{~mm} \times$ $20 \mu \mathrm{m}$, Restek, USA) and a thermal conductivity detector. The operating temperatures of the oven, injector, and detector were $50^{\circ} \mathrm{C}, 100^{\circ} \mathrm{C}$, and $150^{\circ} \mathrm{C}$, respectively, with $\mathrm{He}$ as the carrier gas.

\section{DNA Extraction}

For DNA extraction, 1-ml samples were transferred to $1.5-\mathrm{ml}$ microtubes. Microtubes were centrifuged at $15,000 \times g$ for $5 \mathrm{~min}$, and the supernatants were discarded. All samples were stored at $-70^{\circ} \mathrm{C}$ prior to use. DNA was extracted using the NucleoSpin Soil Kit (Macherey-Nagel, Germany) according to the manufacturer's recommendations. DNA was eluted in $50 \mu \mathrm{l}$ of the elution buffer and quantified using an ASP-2680 spectrophotometer (ACTGene, USA).

\section{RNA Extraction}

RNA was extracted using TRIzol Reagent (Invitrogen, USA) according to the manufacturer's recommendations. RNA was suspended in $50 \mu \mathrm{l}$ of RNase-free water, and the contaminated DNA was removed via DNA digestion. Five $\mu$ l of $10 \times$ DNase I buffer (Ambion, USA) and $2 \mu \mathrm{l}$ of DNase I (Ambion) were added to tubes containing a $50 \mu$ RNA sample. Mixtures were incubated in a water bath at $37^{\circ} \mathrm{C}$ for $30 \mathrm{~min}$. Immediately, RNA was further purified using the RNeasy Mini Kit (Qiagen, USA) according to the manufacturer's recommendations. RNA was eluted in $30 \mu \mathrm{l}$ of the elution buffer and quantified using an ASP-2680 spectrophotometer. RNA was reverse-transcribed using the Omniscript RT Kit (Qiagen) according to the manufacturer's recommendations. Reverse transcription reactions were performed in $20-\mu 1$ volumes. The reaction mixture consisted of $1 \mu \mathrm{l}$ of $1 \times$ buffer RT, $2 \mu \mathrm{l}$ of dNTP, random hexamer at $50 \mu \mathrm{M}, 10 \mathrm{U}$ of RNase inhibitor, $1 \mu \mathrm{l}$ of Omniscript RT, and $10 \mu \mathrm{l}$ of template RNA.

\section{Real-Time PCR}

DNA and transcript level of pMMO quantification was performed using an Applied Biosystems 7300 real-time PCR system (Applied Biosystems, USA). The primer set was used to quantify the M6 populations targeting the 16S rRNA gene: M6-F (5'-CGGAATCAC TGGGCGTAAA-3') and M6-R (5'-GACTCGAGACCTCCAGTATCA$\left.3^{\prime}\right)$ [21]. A primer set of pmoA-F (5'-TTCTGGTGGGTGAATTTC CGCCTT-3') and pmoA-R (5'-AAGCAGGATCACGTCAAGCCA GAT-3') was used to quantify the $p m o A$ gene [21], which encodes the $\alpha$-subunit of the particulate methane monooxygenase. We confirmed that the M6 primer set exclusively amplifies its own target DNA. We designed the following primer set to exclusively 
amplify the NM2 over M6 [26, 27]: NM2-F (5'-CCGTAACTGACG CTGAGGAG- ${ }^{\prime}$ ) and NM2-R (5'-GTGGAATGGTCCCCACAAC$3^{\prime}$ ) targeting the $16 \mathrm{~S}$ rRNA gene. The qPCR was performed with $25-\mu \mathrm{l}$ reaction volumes. The reaction mixture consisted of $12.5 \mu \mathrm{l}$ of PCR premix (Qiagen), $0.5 \mu \mathrm{l}$ of forward primer $(10 \mu \mathrm{M}), 0.5 \mu \mathrm{l}$ of reverse primer $(10 \mu \mathrm{M})$, and $2 \mu \mathrm{l}$ of template DNA. Control reactions contained the same mixtures but with $2 \mu \mathrm{l}$ of ultrapure water replacing the DNA template. The $\mathrm{PCR}$ was initiated at $95^{\circ} \mathrm{C}$ for $15 \mathrm{~min}$, followed by 40 cycles of $94^{\circ} \mathrm{C}$ for $15 \mathrm{sec}$ and $60^{\circ} \mathrm{C}$ for $1 \mathrm{~min}$. The M6 and pmoA sets were used to quantify the transcripts of the 16S rRNA (as an internal standard) and pmoA (as a target) genes, and relative expression (RE) of the pMMO gene was calculated by applying the $\Delta \Delta \mathrm{Ct}$ method as $\mathrm{RE}=2^{-(\Delta \mathrm{Ct}(\text { (co-culture }) \Delta \mathrm{Ct}(\text { control) })}$ where $\Delta \mathrm{Ct}$ is $\mathrm{Ct}_{\mathrm{pmoA}}-\mathrm{Ct}_{16 \mathrm{~S} \text { rRNA }}$.

\section{Results}

\section{Effects of NM2 on Methane Oxidation Activity of Methanotrophs}

We confirmed that NM2 does not utilize methane, methanol, and formate (data not shown). A physiological profile result showed that NM2 utilizes $\alpha$-cyclodextrin, N-acetyl-D-glucosamine, D-mannitol, and D-cellobiose. NM2 showed positive effects on methane oxidation of methanotrophs including Methylocystis sp. M6, Methylosinus trichosporium OB3b, and the methanotrophic consortia A and B (Fig. 1). Addition of NM2 significantly increased the methane removal in co-cultures $(p<0.05)$, except for consortium A. The methane removal rates were $9.24 \pm 0.02$ and $16.9 \pm 0.45 \mu \mathrm{mol} \cdot \mathrm{l}^{-1} \cdot \mathrm{d}^{-1}$ in M6 alone and NM2-M6 coculture, respectively ( $82.9 \%$ increase) (Fig. 1A), $8.55 \pm 0.26$ and $20.3 \pm 0.07 \mu \mathrm{mol} \cdot \mathrm{l}^{-1} \cdot \mathrm{d}^{-1}$ in OB3b alone and NM2-OB3b co-culture, respectively (137.4\% increase) (Fig. 1B), $10.16 \pm$
0.31 and $15.25 \pm 2.71 \mu \mathrm{mol} \cdot \mathrm{l}^{-1} \cdot \mathrm{d}^{-1}$ in consortium A alone and co-culture of NM2-consortium A, respectively $(50.1 \%$ increase) (Fig. 1C), and $9.39 \pm 0.24$ and $15.9 \pm 0.18 \mu \mathrm{mol} \cdot \mathrm{l}^{-1} \cdot \mathrm{d}^{-1}$ in consortium B alone and co-culture of NM2-consortium B, respectively (69.3\% increase) (Fig. 1D). Enhancement of methanotrophic activity by NM2 was more efficient with the isolates than with the consortia $(82.9 \%$ and $137.4 \%$ vs. $50.1 \%$ and $69.3 \%$, respectively).

\section{Effects of NM2 on Growth and Activity of M6}

Effects of NM2 on methanotrophic activity and growth of M6 are shown in Fig. 2. Cell mass peaked at $1.2 \pm 0.1 \mathrm{~g} / 1$ on day 14 in NM2-M6 co-culture. Cell mass increased to $0.4 \pm$ $0.1 \mathrm{~g} / 1$ by the end of the 21-day period in M6 alone (Fig. 2A). The amounts of cumulative methane removal at the end of the period were $13.0 \pm 3.2$ and $2.5 \pm 0.2 \mathrm{mmol}$ in the coculture and M6 alone, respectively (Fig. 2B). NM2 increased the cell growth and methanotrophic activity of M6 by three and five times, respectively.

M6 population rapidly increased for the first 7 days, and then almost leveled off, reaching $2.5 \times 10^{12} \pm 1.9 \times 10^{11}$ and $4.4 \times 10^{11} \pm 1.1 \times 10^{10} 16 \mathrm{~S}$ rDNA copy number $\cdot l^{-1}$ in the coculture and M6 alone, respectively, by the end of the experimental period. The relative expression levels of the pmo $A$ gene were $7.7 \pm 0.9$ and $1.7 \pm 1.5$ at 7 and 14 days, respectively (Fig. $2 \mathrm{C}$ ). The results revealed that the rapid increase of methane removal was positively correlated with the $p m o A$ gene expression.

Unexpectedly, NM2 population sharply decreased by one order of magnitude with time, while the theoretical population level of NM2 only considering dilution, derived by subtracting the cell mass that flowed out of culture,

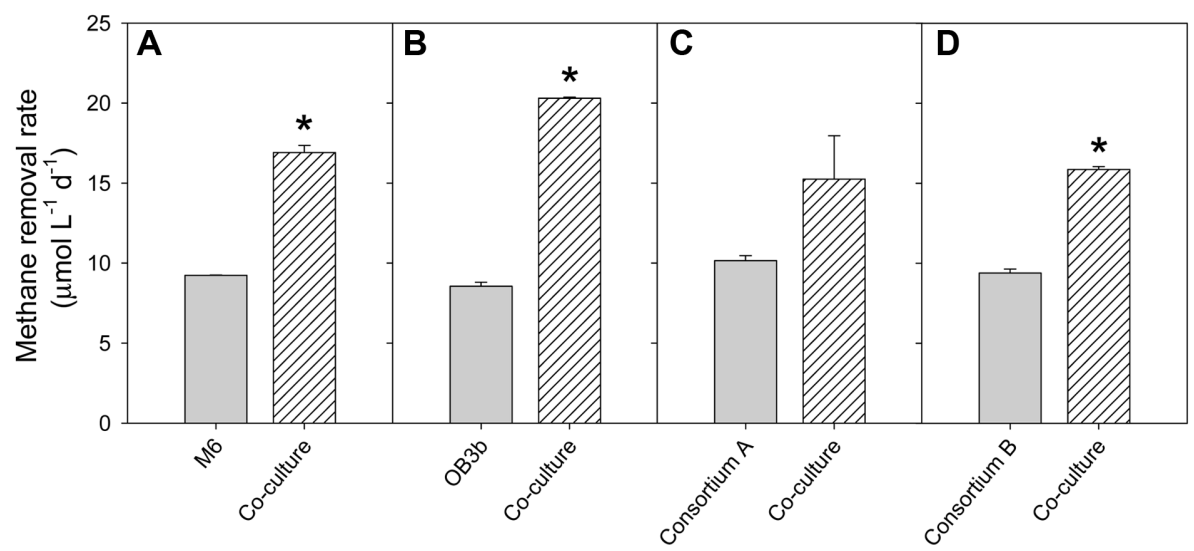

Fig. 1. Effects of Microbacterium sp. NM2 on methanotrophic activity of different methanotrophs, including Methylocystis sp. M6 (A), Methylosinus trichosporium OB3b (B), methanotrophic consortium A (C), and methanotrophic consortium B (D). The symbol * indicates a significant difference $(p<0.05)$. Error bars indicate the standard deviation of the mean. 
A

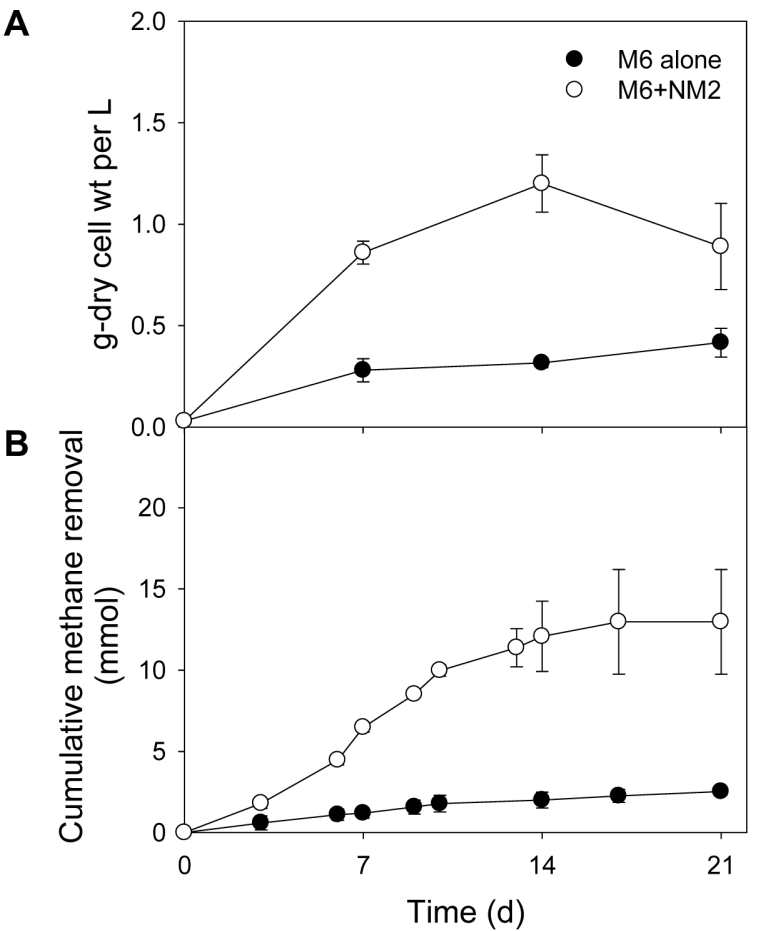

C

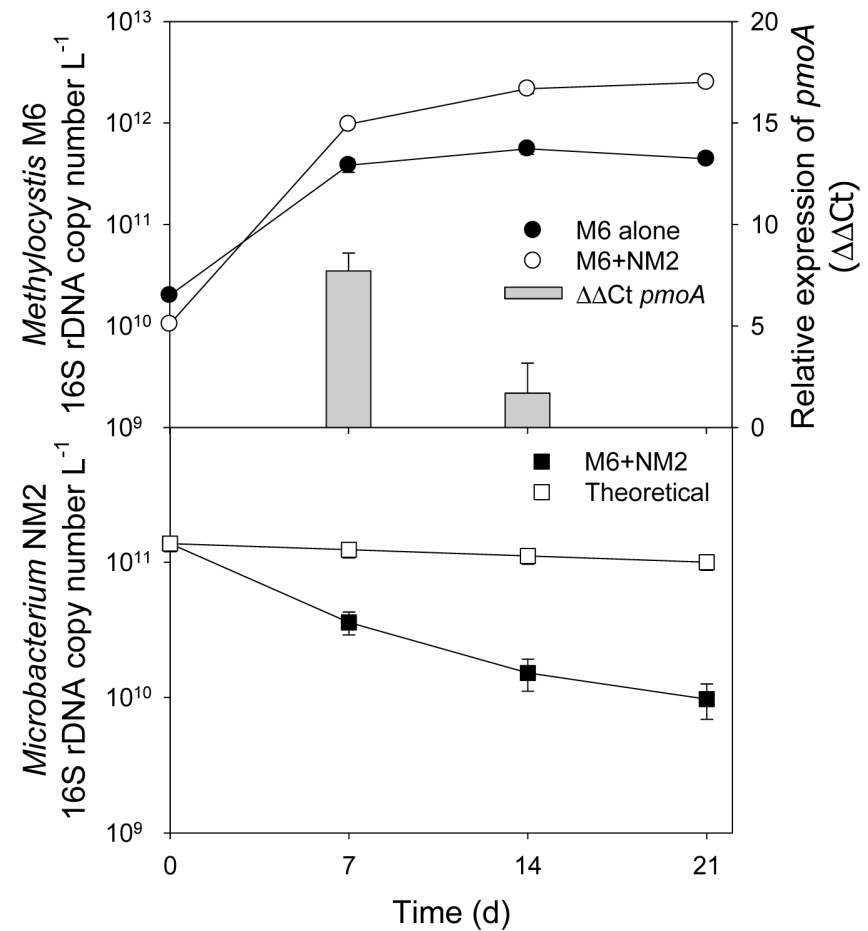

Fig. 2. Effects of Microbacterium sp. NM2 on activity and growth of Methylocystis sp. M6. Cell mass (A), methane removal (B), M6 population and relative expression of pmoA (C), and NM2 population (D).

Ten $\mathrm{ml}$ of the microbial cultures $(10 \%)$ were replaced with an equal volume of fresh medium every 7 days. Theoretical population level is the population density of NM2 derived by subtracting cell mass that flowed out of culture. Error bars indicate the standard deviation of the mean.

appeared to remain stable (Fig. 2D). In contrast, the M6 population increased by two orders of magnitude (Fig. 2C). Thus, a majority of the cell mass originated from M6 growth in the co-culture. A linearity test showed that the masses of the two organisms had a negative relationship $\left(R^{2}=0.90\right)$. These results indicate that M6 could adversely influence the growth and survival of NM2.

\section{Interaction of M6 and NM2 when No Carbons Were Supplied}

Interaction between M6 and NM2 in the absence of carbon was identified (Fig. 3). Without a carbon supply, M6 population markedly decreased (by $85 \%$ ) within the first 7 days in M6 alone, but M6 population remained stable at the initial level over time when co-cultured with NM2 (Fig. 3A). The NM2 population was stable at the initial level for at least 21 days in the absence of a carbon supply in NM2 alone (Fig. 3B). However, when NM2 was co-cultured with M6, NM2 population decreased by $66 \%$ at the end of the experimental period. These results indicate that M6 can take advantage of NM2 for survival under starvation conditions.

\section{Effects of Physical Contact in the Co-Culture}

A dialysis membrane (pore size of $50 \mathrm{kDa}$ equivalent to 50,000 molecular weight) was used to determine whether enhancement of M6 activity by NM2 is mediated by an exchange of chemical compounds or by a physical interaction (Fig. 4). The pore size is comparable to that of a bacterial cysteine proteinase [28], and much larger than the compounds reported as stimulators of methanotrophic activity, e.g., cobalamin $(1.3 \mathrm{kDa})$, riboflavin $(0.3 \mathrm{kDa})$ and organic acids $(0.1 \mathrm{kDa})[17,29]$. We observed that a heterotrophic bacterium within the same tube was active and grew under the same experimental settings for more than 90 days (data not shown), indicating that NM2 was presumably active within the tube for this 4-day experimental period. The amounts of methane removed were approximately 100,000 ppm after 4 days in M6 alone and in a co-culture system in which M6 and NM2 were physically separated by a dialysis membrane, whereas approximately 200,000 ppm of methane were removed in a co-culture system in which M6 and NM2 were mixed together. Physical contact of NM2 with M6 enhanced the methanotrophic activity of M6 by about two 


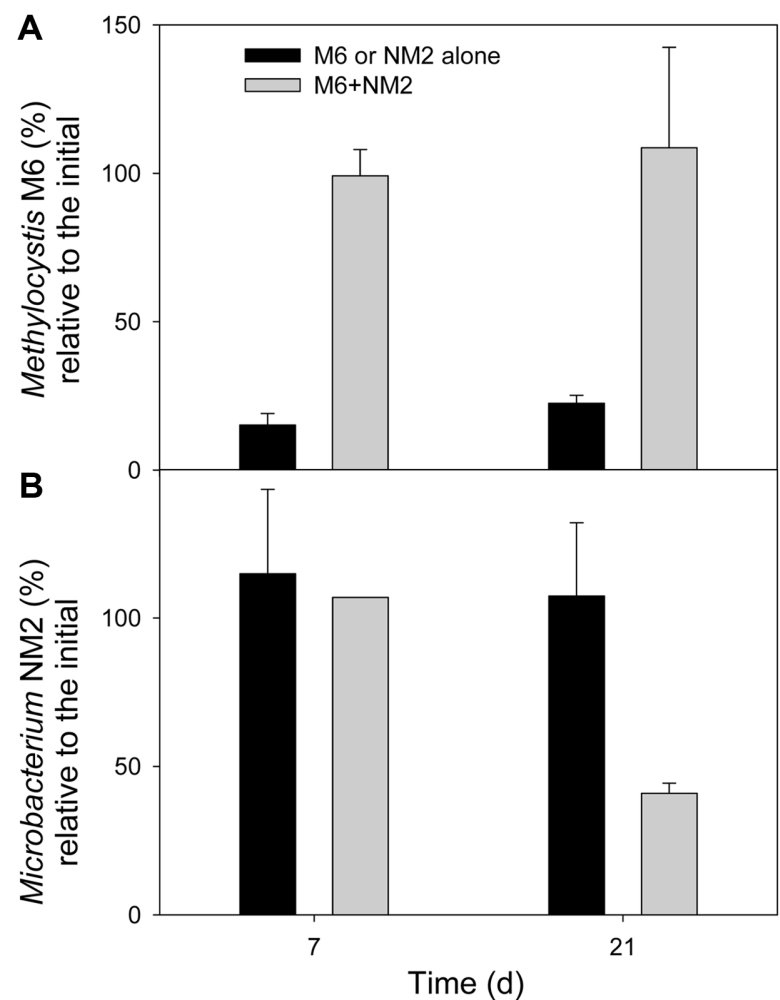

Fig. 3. Survival of Microbacterium sp. NM2 and Methylocystis sp. M6 in the absence of carbon supply.

M6 (A) and NM2 (B) populations. Error bars indicate the standard deviation of the mean.

times, compared with that in the co-culture system with a dialysis membrane. The physical contact apparently reduced the lag period for methane oxidation. These results indicate that the physical separation of NM2 from M6 cannot enhance the methanotrophic activity, and that physical contact is required for their interaction.

\section{NM2 Effects on Growth and Activity of M6 under a Fed- Batch Condition}

Biomass, methane removal, and microbial populations were monitored in a fed-batch culture with a HRT of 5 days (Fig. 5). Cell mass and methane removal rate were stably maintained in NM2-M6 co-culture after an acclimation period of 6 days. Cell mass in the co-culture increased to $1.1 \mathrm{~g} / 1$ for the acclimation period and remained at $1.02 \pm$ $0.1 \mathrm{~g} / 1$ during the 16-day experimental period. Methane removal rate in the co-culture rapidly increased to $38.1 \mu \mathrm{mol} \cdot \mathrm{l}^{-1} \cdot \mathrm{d}^{-1}$ during the acclimation period, and the removal rate during the experiment period was $28.4 \pm$ $5.4 \mu \mathrm{mol} \cdot \mathrm{l}^{-1} \cdot \mathrm{d}^{-1}$. After an acclimation period, cell mass of the M6 alone remained at $0.92 \pm 0.1 \mathrm{~g} / 1$ for the experimental

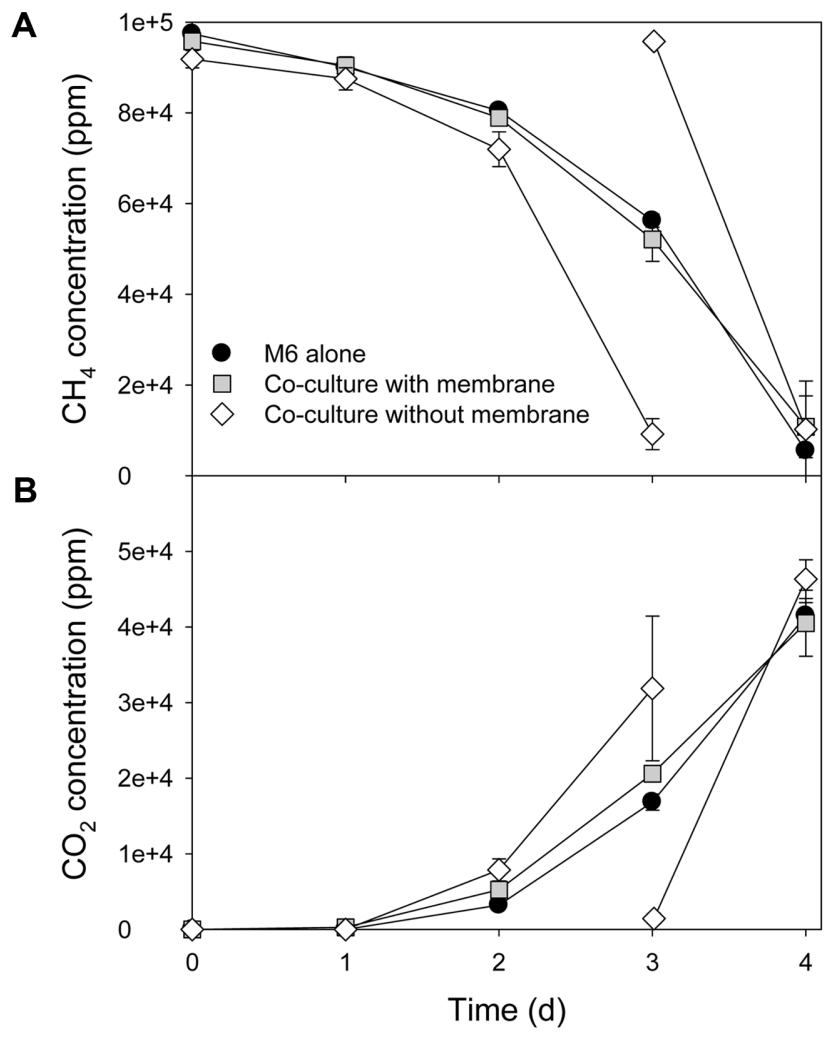

Fig. 4. Effects of physical contact of Methylocystis sp. M6 and Microbacterium sp. NM2 on methane oxidation (A) and $\mathrm{CO}_{2}$ production (B).

When methane concentration was below 10,000 ppm, methane was spiked again into the bottle. Error bars indicate the standard deviation of the mean.

period, while methane removal rate was reduced by day 6 and then maintained at $13.5 \pm 2.9 \mu \mathrm{mol} \cdot \mathrm{l}^{-1} \cdot \mathrm{d}^{-1}$ during the acclimation period $\left(18.8 \pm 9.3 \mu \mathrm{mol} \cdot \mathrm{l}^{-1} \cdot \mathrm{d}^{-1}\right.$ for the 16-day experimental period).

M6 population rapidly increased for the acclimation period, and then was stable at $1.1 \times 10^{11} \pm 1.8 \times 10^{10} 16 \mathrm{~S}$ rDNA copy number $\mathrm{l}^{-1}$ over time, whereas NM2 population slightly increased during the acclimation period, and then gradually decreased (Fig. 5C). When theoretical NM2 population levels were estimated by subtracting the cells that flowed out, it appeared that the reduction resulted from the washing-out of NM2 cells in the fed-batch process. These results indicate that M6 does not negatively influence NM2 when it actively proliferates with methane.

\section{Discussion}

In this study, we investigated the interaction between 
A

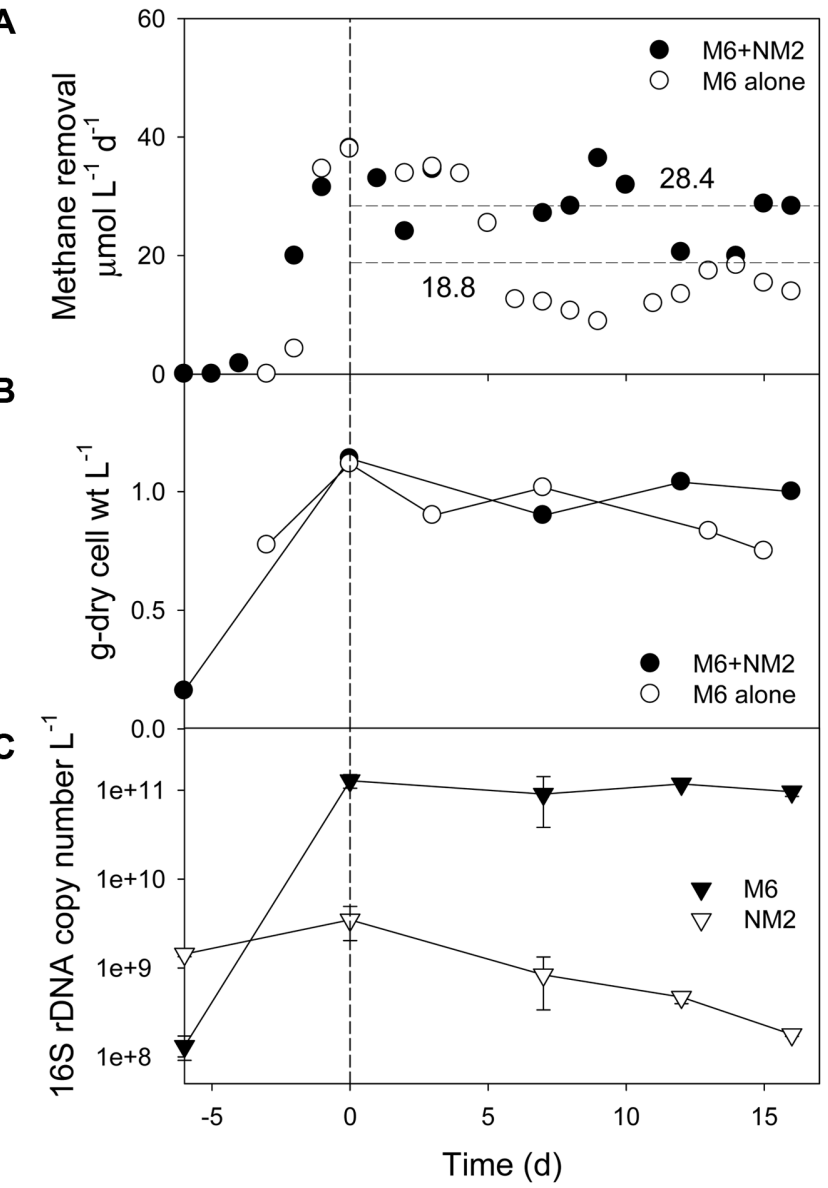

Fig. 5. Effects of Microbacterium sp. NM2 on biomass growth, methanotrophic activity, and population dynamics of Methylocystis sp. M6 at a hydraulic retention time of 5 days.

Methane removal in a M6-NM2 co-culture and M6 alone (A), cell mass in the co-culture and M6 alone (B), populations of M6 and NM2 in the co-culture $(\mathbf{C})$. Error bars indicate the standard deviation of the mean.

the non-methylotroph Microbacterium sp. NM2 and the methanotroph Methylocystis sp. M6. Microbacterium spp. (Gram-positive actinomycetes) and Methylocystis spp. (Gramnegative proteobacteria) are found in diverse environments. Members of the genus Microbacterium are reported to be a decomposer in soil and activated sludge [30-32]. NM2 utilizes multi-carbon compounds as substrates, but does not utilize $\mathrm{C} 1$ compounds, such as methane, methanol, and formate. Consistently, its draft genome indicates that it lacks the genes related with the oxidation of methane, methanol and formate (data not shown). NM2 enhanced the methane-oxidizing activity of methanotrophs including $\mathrm{M} 6$ and OB3b as well as the methanotrophic consortia A and B (Fig. 1). Batch co-culture of NM2 and M6 showed that NM2 rapidly increased the biomass and M6 population for the first 7 days, as well as the pMMO enzyme expression, resulting in increases of more than 3 -fold in biomass and activity (Fig. 2). These results are similar to those in previous studies reporting that a number of non-methylotrophic bacterial genera such as Rhizobium, Flavobacterium, Sphingopyxis, and Cupriavidus can enhance the activity and growth of specific methanotrophic isolates when they are cultivated together [17, 18, 21]. In addition, diversity of heterotrophs was correlated with the methanotrophic activity [33]. The enhancement of methanotrophic activity by NM2 probably begins with activation of the $\mathrm{PMMO}$ gene of M6, by which the M6 population is increased. The increases in population and $\mathrm{pMMO}$ gene expression are responsible for the activity enhancement because methanotrophic activity is positively correlated with the biomass of methanotrophs [17, 34, 35]. Similarly, Sphingopyxis sp. NM1 enhanced the transcriptional gene expression involved in methane oxidation, resulting in enhancement of the activity and growth of M6 [21]. Moreover, the fed-batch results indicate that the co-culture was more efficient in terms of methane removal than the pure culture (28.4 vs. $\left.18.8 \mu \mathrm{mol} \cdot \mathrm{l}^{-1} \cdot \mathrm{d}^{-1}\right)$ although there were similar biomass levels in the two culture types (Fig. 5), suggesting that the presence of the non-methylotroph may increase cell-specific activity of methanotrophs. Therefore, NM2 can act as a biological agent for the enhancement of methanotrophic systems or as a helper microorganism in the growth of methanotrophs.

In general, the exchange of organic compounds is supposed to be a key mechanism responsible for enhancement of methanotrophic growth and activity by non-methylotrophs. It has been reported that chemical compounds originating from non-methylotrophic isolates enhance the growth and activity of the co-cultured methanotrophs [17, 29]. For instance, cobalamin from a Rhizobium isolate stimulated the methanotrophic activities of methanotrophs belonging to Methylococcaceae, Methylomonas, and Methylobacter [17], while riboflavin and organic acids resulted in an increase in Methylosinus growth [29]. However, our dialysis membrane experiment showed that physical contact between NM2 and M6 was required to stimulate the methanotrophic activity and growth of M6 (Fig. 4). Therefore, we speculate that M6 may obtain growth/activity-promoting compounds from NM2 through direct contact. We were unable to find an inter-bacteria interaction study that included results comparable with ours; however, there was an observation reported by Schroeckh et al. [36] indicating that an intimate physical interaction between microorganisms coerced a participant into doing a particular function, e.g., the 
production of aromatic polyketides. Ho et al. [33] reported that methanotrophic activity was stimulated when the methanotroph was co-cultured with non-methanotrophic heterotrophs, but not when it was grown in nonmethanotroph-spent medium. Previously, we performed another dialysis membrane experiment with M6 and Sphingopyxis sp. NM1, a biological stimulator for M6 [21], and observed that there was no need of physical proximity for growth/activity enhancement (data not shown). Veraart et al. [37] reported that methanotrophs may be able to interact with heterotrophic bacteria, mediated by volatile compounds. These observations indicate that methanotrophs can take advantage of other non-methylotrophic bacteria with or without physical contact. The requirement of physical contact can indicate the higher level of specificity against the counterpart for obtaining growth/activity-promoting substrates, compared with non-physical interactions. This physical intimacy may result in better efficiency and specificity for a methanotroph in obtaining benefits from its counterparts in natural environments that are highly complexed and densely populated with different microorganisms.

Methanotrophs can be associated with biotic components within natural environments in several different ways. For example, they have been reported to interact with many different adjacent organisms such as plants, protists, and marine invertebrates as well as with other bacteria [1, 2]; e.g., predator-prey relationships with zooplankton and protozoa [11,38], mutualistic relationships with mussels and invertebrates [10], synergistic relationships with mosses and algae [12-14], and commensal relationships with nonmethylotrophic bacteria $[17,21]$. In this study, we observed a particular relationship between a methanotroph and a non-methylotrophic bacterium in a series of results (Figs. 24). First of all, the dialysis membrane results indicated that physical contact is a prerequisite for the enhancement of M6 activity and growth (Fig. 4). The starvation assay results showed that NM2 population decreased by $66 \%$ while the M6 population remained stable during the 21-day co-culture period, and that NM2 population remained at the initial level while M6 population decreased by $85 \%$ in the eitherone-alone test (Fig. 3). Moreover, M6 took advantage of NM2 for survival under starvation conditions, from which we speculated that M6 can extort growth substrates from NM2. In addition, the batch co-culture showed that the M6 population increased by two orders of magnitude, while the NM2 population sharply decreased by one order of magnitude (Fig. 2). The comparison between the actual and expected population levels of NM2 indicated that M6 was responsible for the reduction of NM2 population. In contrast, the fed-batch co-culture showed that M6 population was stably maintained at the initial level while NM2 population gradually decreased (Fig. 5), a result of the washing-out of NM2 cells in the fed-batch process. These results indicate that M6 does not negatively affect the survival of NM2 when it is actively proliferating with methane (Figs. 2 and 5).

Five types of inter-microorganism interactions, such as commensalism, synergism, mutualism, parasitism, and predation, can be beneficial to at least one participant. Mutualism, parasitism, and predation require an intimate physical interaction. For example, Trichoderma spp. have been shown to be parasitic against a broad range of filamentous fungi, although they are normally saprophytic in soil [39]. Members in the bacterial genus Bdellovibrio are parasitic against other gram-negative bacteria [40]. These parasites must be in contact with the target in order to initiate their parasitic interaction. The observed interaction between M6 and NM2 is beneficial to the methanotroph, but unfavorable to the non-methylotroph through direct physical contact in a specific situation, seemingly parasitism, although methanotrophs have never been suspected as parasites. Most methanotrophs have been shown to use methane as their sole carbon and energy source as obligate methanotrophs. It should be pointed out that the results in the present study have been obtained under conventional nutrient and cultivation conditions in laboratories. Thus, it is possible that methanotrophs can use alternative substrates as growth- and/or activity-promoting substrates, such as those originating from other bacteria in natural environments.

In conclusion, the non-methylotroph Microbacterium sp. NM2 can enhance the methane oxidation activity of methanotrophs including the methanotroph Methylocystis sp. M6 because it can stimulate the population growth and increase the expression of pMMO. The NM2 and M6 coculture experiments indicate that NM2 is effective in such enhancement only when NM2 is in physical contact with M6, and that the interaction is positive to M6 but negative to NM2. The requirement of physical proximity suggests a tight association between methanotrophs and nonmethylotrophs in natural environments. To elucidate the relationship between M6 and NM2, further mechanistic investigations into the exchange of resources, signaling, and physical interactions are required. In addition, a further study on the direct contact using high-resolution microscopy is needed. However, the current results provide a significant insight into the survival and growth of methanotrophs in natural soil environments. 


\section{Acknowledgments}

This research was supported by the Basic Science Research Program through the National Research Foundation of Korea funded by the Ministry of Education (2018R1D1A1B07048872).

\section{Conflict of Interest}

The authors have no financial conflicts of interest to declare.

\section{References}

1. Hanson RS, Hanson TE. 1996. Methanotrophic bacteria. Microbiol. Rev. 60: 439-471.

2. Semrau JD, DiSpirito AA, Yoon S. 2010. Methanotrophs and copper. FEMS Microbiol. Rev. 34: 496-531.

3. Fox BG, Borneman JG, Wackett LP, Lipscomb JD. 1990. Haloalkene oxidation by the soluble methane monooxygenase from Methylosinus trichosporium OB3b: mechanistic and environmental implications. Biochemistry 29: 6419-6427.

4. Burrows KJ, Cornish A, Scott D, Higgins IJ. 1984. Substrate specificities of the soluble and particulate methane monooxygenases of Methylosinus trichosporium OB3b. Microbiology 130: 3327-3333.

5. Colby J, Stirling DI, Dalton H. 1977. The soluble methane mono-oxygenase of Methylococcus capsulatus (Bath). Its ability to oxygenate n-alkanes, n-alkenes, ethers, and alicyclic, aromatic and heterocyclic compounds. Biochem. J. 165: 395-402.

6. Jiang $H$, Chen $Y$, Jiang $P$, Zhang C, Smith TJ, Murrell JC, et al. 2010. Methanotrophs: Multifunctional bacteria with promising applications in environmental bioengineering. Biochem. Eng. J. 49: 277-288.

7. Huber-Humer M, Gebert J, Hilger H. 2008. Biotic systems to mitigate landfill methane emissions. Waste Manag. Res. 26: 33-46.

8. Dedysh SN, Knief C, Dunfield PF. 2005. Methylocella species are facultatively methanotrophic. J. Bacteriol. 187: 4665-4670.

9. Knief C. 2015. Diversity and habitat preferences of cultivated and uncultivated aerobic methanotrophic bacteria evaluated based on pmoA as molecular marker. Front Microbiol. 6: 1346.

10. Petersen JM, Dubilier N. 2009. Methanotrophic symbioses in marine invertebrates. Environ. Microbiol. Rep. 1: 319-335.

11. Murase J, Frenzel P. 2008. Selective grazing of methanotrophs by protozoa in a rice field soil. FEMS Microbiol. Ecol. 65: 408-414.

12. Kip N, Van Winden JF, Pan Y, Bodrossy L, Reichart G-J, Smolders AJ, et al. 2010. Global prevalence of methane oxidation by symbiotic bacteria in peat-moss ecosystems. Nat. Geosci. 3: 617-621.

13. Raghoebarsing AA, Smolders AJP, Schmid MC, Rijpstra WIC, Wolters-Arts M, Derksen J, et al. 2005. Methanotrophic symbionts provide carbon for photosynthesis in peat bogs. Nature 436: 1153-1156.

14. Van der Ha D, Bundervoet B, Verstraete W, Boon N. 2011. A sustainable, carbon neutral methane oxidation by a partnership of methane oxidizing communities and microalgae. Water Res. 45: 2845-2854.

15. Gonzalez JM, Sherr EB, Sherr BF. 1990. Size-selective grazing on bacteria by natural assemblages of estuarine flagellates and ciliates. Appl. Environ. Microbiol. 56: 583-589.

16. Atlas RM, Bartha R. 1997. Microbial ecology: fundamentals and applications, pp. 4 Ed. Benjamin/Cummings Science Publishing, Melon Park.

17. Iguchi H, Yurimoto H, Sakai Y. 2011. Stimulation of methanotrophic growth in cocultures by cobalamin excreted by rhizobia. Appl. Environ. Microbiol. 77: 8509-8515.

18. Stock M, Hoefman S, Kerckhof F-M, Boon N, De Vos P, De Baets B, et al. 2013. Exploration and prediction of interactions between methanotrophs and heterotrophs. Res. Microbiol. 164: 1045-1054.

19. Hršak D, Begonja A. 1998. Growth characteristics and metabolic activities of the methanotrophic-heterotrophic groundwater community. J. Appl. Microbiol. 85: 448-456.

20. Wilkinson TG, Topiwala H, Hamer G. 1974. Interactions in a mixed bacterial population growing on methane in continuous culture. Biotechnol. Bioeng. 16: 41-59.

21. Jeong S-Y, Cho K-S, Kim TG. 2014. Density-dependent enhancement of methane oxidation activity and growth of methylocystis sp. by a non-methanotrophic bacterium Sphingopyxis sp. Biotechnol. Rep. 4: 128-133.

22. Whittenbury R, Phillips KC, Wilkinson JF. 1970. Enrichment, isolation and some properties of methane-utilizing bacteria. J. Gen. Microbiol. 61: 205-218.

23. Lee E-H, Yi T, Moon K-E, Park H, Ryu HW, Cho K-S. 2011. Characterization of methane oxidation by a methanotroph isolated from a landfill cover soil, south Korea. J. Microbiol. Biotechnol. 21: 753-756.

24. Lee E-H, Park H, Cho K-S. 2010. Characterization of methane, benzene and toluene-oxidizing consortia enriched from landfill and riparian wetland soils. J. Hazard. Mater. 184: 313-320.

25. Kim TG, Yi T, Lee E-H, Ryu HW, Cho K-S. 2012. Characterization of a methane-oxidizing biofilm using microarray, and confocal microscopy with image and geostatic analyses. Appl. Microbiol. Biotech. 95: 1051-1059.

26. Kim TG, Jeong S-Y, Cho K-S. 2015. Development of droplet digital PCR assays for methanogenic taxa and examination of methanogen communities in full-scale anaerobic digesters. Appl. Microbiol. Biotech. 99: 445-458.

27. Kim TG, Jeong S-Y, Cho K-S. 2014. Comparison of droplet digital PCR and quantitative real-time PCR for examining population dynamics of bacteria in soil. Appl. Microbiol. Biotech. 98: 6105-6113.

28. Chen Z, Potempa J, Polanowski A, Wikstrom M, Travis J. 1992. Purification and characterization of a 50-kDa cysteine 
proteinase (gingipain) from Porphyromonas gingivalis. J. Biol. Chem. 267: 18896-18901.

29. Xing X-H, Wu H, Luo M-F, Wang B-P. 2006. Effects of organic chemicals on growth of Methylosinus trichosporium OB3b. Biochem. Eng. J. 31: 113-117.

30. Manickam N, Mau M, Schlömann M. 2006. Characterization of the novel HCH-degrading strain, Microbacterium sp. ITRC1. Appl. Microbiol. Biotech. 69: 580-588.

31. Sheng X, He L, Zhou L, Shen Y. 2009. Characterization of Microbacterium sp. F10a and its role in polycyclic aromatic hydrocarbon removal in low-temperature soil. Can. J. Microbiol. 55: 529-535.

32. Chen J-A, Li X, Li J, Cao J, Qiu Z, Zhao Q, et al. 2007. Degradation of environmental endocrine disruptor di-2ethylhexyl phthalate by a newly discovered bacterium, Microbacterium sp. strain CQ0110Y. Appl. Microbiol. Biotech. 74: $676-682$.

33. Ho A, De Roy K, Thas O, De Neve J, Hoefman S, Vandamme P, et al. 2014. The more, the merrier: heterotroph richness stimulates methanotrophic activity. ISME J. 8: 1945-1948.

34. Kim TG, Yi T, Yun J, Ryu HW, Cho K-S. 2013. Biodegradation capacity utilization as a new index for evaluating biodegradation rate of methane. J. Microbiol. Biotechnol. 23: 715-718.

35. Dianou D, Adachi K. 1999. Characterization of methanotrophic bacteria isolated from a subtropical paddy field. FEMS Microbiol. Lett. 173: 163-173.

36. Schroeckh V, Scherlach K, Nützmann H-W, Shelest E, Schmidt-Heck W, Schuemann J, et al. 2009. Intimate bacterialfungal interaction triggers biosynthesis of archetypal polyketides in Aspergillus nidulans. Proc. Natl. Acad. Sci. USA 106: 14558-14563.

37. Veraart A, Garbeva P, Beersum F, Ho A, Hordijk C, MeimaFranke $\mathrm{M}$, et al. 2018. Living apart together-bacterial volatiles influence methanotrophic growth and activity. ISME J. 12: 1163-1166.

38. Kankaala P, Huotari J, Peltomaa E, Saloranta T, Ojala A. 2006. Methanotrophic activity in relation to methane efflux and total heterotrophic bacterial production in a stratified, humic, boreal lake. Limnol. Oceanogr. 51: 1195-1204.

39. Samuels GJ. 1996. Trichoderma: a review of biology and systematics of the genus. Mycological Research. 8: 923-935.

40. Stolp H. 1973. The bdellovibrios: Bacterial parasites of bacteria. Annu. Rev. Phytopathol. 11: 53-76. 